\title{
Neuroprotective Effect of Xueshuantong for Injection (Lyophilized) in Transient and Permanent Rat Cerebral Ischemia Model
}

\author{
Xumei Wang, ${ }^{1}$ Shaoxia Wang, ${ }^{1}$ Jinxin Wang, ${ }^{1}$ Hong Guo, ${ }^{2}$ Zhaopeng Dong, ${ }^{3}$ \\ Lijuan Chai, ${ }^{2}$ Limin $\mathrm{Hu}^{2}{ }^{2}$ Yue Zhang, ${ }^{2}$ Hong Wang, ${ }^{1}$ and Lu Chen ${ }^{1}$ \\ ${ }^{1}$ Tianjin State Key Laboratory of Modern Chinese Medicine, Tianjin University of Traditional Chinese Medicine, \\ Tianjin 300193, China \\ ${ }^{2}$ Tianjin Key Laboratory of Chinese Medicine Pharmacology, Tianjin University of Traditional Chinese Medicine, \\ Tianjin 300193, China \\ ${ }^{3}$ Zhongnuo R\&D Department, CSPC Zhongnuo Pharmaceutical (Shijiazhuang) Co., Ltd., Hebei 050051, China
}

Correspondence should be addressed to Shaoxia Wang; wangshaoxia1978@hotmail.com and Limin Hu; huliminth@126.com

Received 30 July 2015; Revised 2 November 2015; Accepted 8 November 2015

Academic Editor: Chang-Gue Son

Copyright (c) 2015 Xumei Wang et al. This is an open access article distributed under the Creative Commons Attribution License, which permits unrestricted use, distribution, and reproduction in any medium, provided the original work is properly cited.

\begin{abstract}
Xueshuantong for Injection (Lyophilized) (XST), a Chinese Materia Medica standardized product extracted from Panax notoginseng (Burk.), is used extensively for the treatment of cerebrovascular diseases such as acutely cerebral infarction clinically in China. In the present study, we evaluated the acute and extended protective effects of XST in different rat cerebral ischemic model and explored its effect on peroxiredoxin (Prx) 6-toll-like receptor (TLR) 4 signaling pathway. We found that XST treatment for 3 days could significantly inhibit transient middle cerebral artery occlusion (MCAO) induced infarct volume and swelling percent and regulate the mRNA expression of interleukin-1 $\beta$ (IL-1 $\beta$ ), IL-17, IL-23p19, tumor necrosis factor- $\alpha$ (TNF $\alpha$ ), and inducible nitric oxide synthase (iNOS) in brain. Further study demonstrated that treatment with XST suppressed the protein expression of peroxiredoxin (Prx) 6-toll-like receptor (TLR) 4 and phosphorylation level of p38 and upregulated the phosphorylation level of STAT3. In permanent MCAO rats, XST could reduce the infarct volume and swelling percent. Moreover, our results revealed that XST treatment could increase the rats' weight and improve a batch of functional outcomes. In conclusion, the present data suggested that XST could protect against ischemia injury in transient and permanent MCAO rats, which might be related to Prx6-TLR4 pathway.
\end{abstract}

\section{Introduction}

Stroke is harmful to human health and has a high incidence. It is a leading cause of death and the third cause of disability. The loss of neurological functions following stroke is caused by massive loss of neurons resulting from ischemic insults. Up to now, tissue plasminogen activators (tPAs) are still the only agents approved by the Food and Drug Administration since 1996 [1]. However, tPA is currently used in fewer than $5 \%$ of stroke victims [2], because of its narrow therapeutic window and risks of cerebral hemorrhage [3]. Therefore, the development of a new drug for stroke with increasing efficacy remains an urgent priority.

More and more evidence suggests that immunomodulation plays an irreplaceable role in postischemic neuroinflammation, which could be a consequence of toll-like receptor (TLR) activation [4]. And the blockade of TLR4 is intimately associated with experimental and clinical outcome in ischemic stroke $[4,5]$. Peroxiredoxin (Prx) 6 is newly identified TLR4-dependent inducers of infiltrating macrophage activation and the subsequent production of inflammatory mediators from invading $\mathrm{T}$ cells in the ischemic brain $[6$, 
7]. Increased Prx6 was reported to be the major danger associated molecular patterns (DAMPs) that induce infiltrating macrophage activation and the subsequent production of cytokines from invading $\mathrm{T}$ cells after cerebral ischemia [8] through TLR4 signal pathway. Therefore, Prx6-TLR4 signaling pathway is believed to be an oval therapeutic strategy for poststroke neuroinflammation and brain injury $[4,9]$.

In contrast with Western developed countries, China has many herbs and herbal standardized preparations for the clinical treatment of ischemic stroke. San-chi, the root of Panax notoginseng (Burk.), is one of the most famous traditional Chinese medicinal herbs. It is widely used to cure heart disease [10], ischemic stroke [11], and acute intracerebral hemorrhage [12] and relieve pain [13] in China or other oriental countries for thousands of years. Panax notoginseng saponins are believed to be main active components of San-chi $[14,15]$. Xueshuantong for Injection (Lyophilized) (XST) is a standardized product extracted from San-chi. It is recorded by the People's Republic of China Pharmacopoeia and is used extensively for the treatment of cardiovascular disease, cerebrovascular disease, and diabetes in China, with total sales over $\$ 700$ million in 2013 [16]. Its ingredients, which are several kinds of Panax notoginseng saponins, are definite and clear (see Section 2.1). For being accepted worldwide, it demands more strict and accurate evidence for its effectiveness and the principles behind it. In the present study, we evaluated the acute and extended protective effects of XST in different rat cerebral ischemic model and explored its effect on Prx6-TLR4 signaling pathway.

\section{Materials and Methods}

2.1. Drug. XST was got from Wuzhou Pharmaceutical Co., Ltd. (Guangxi province, China). The manufacturing technology was taken according to "Pharmacopoeia of China 2010." The rhizome comminution of Panax notoginseng (Burk.) was extracted by $60 \%$ ethanol under reflux for 3 times (3 hours per time). The extracting solution was merged for decompressing concentration till there was no alcohol taste and it was further subjected to a D101 macroporous absorption resin column eluted with water, $80 \%$ ethanol. The $80 \%$ ethanol extract was dried under vacuum to obtain XST. Previous studies have fully studied the main compositions and contents of this preparation [17, 18]. HPLC fingerprint shows that it contains ginsenosides $\operatorname{Rg} 148.1 \%$, ginsenosides Rb1 27.8\%, notoginsenoside R1 11.1\%, ginsenosides Re 5.5\%, ginsenosides $\mathrm{Rd} 1.3 \%$, notoginsenoside $\mathrm{Ra} 1.1 \%$, and $20-\mathrm{O}-$ glucoginsenoside $\mathrm{Rf} 0.7 \%$ [17, 18]. In this study, XST was freshly prepared in $0.9 \%$ normal saline before use.

2.2. Animals. Male healthy Wistar rats $(260 \pm 10 \mathrm{~g})$ were purchased from Vital River Laboratory Animal Technology Co. Ltd. (Beijing, China). The animals were housed under light-controlled conditions, $12 \mathrm{~h}$ light $/ 12 \mathrm{~h}$ dark cycle, and at room temperature of $22^{\circ} \mathrm{C}$. Food and water were available freely. All animal procedures were performed according to the Animal Care and Use Committee at Tianjin University of Traditional Chinese Medicine in China.

2.3. Middle Cerebral Artery Occlusion. Anesthesia was induced with chloral hydrate $(10 \%, 3 \mathrm{~mL} / \mathrm{kg})$. All groups except sham group underwent focal ischemia surgery induced by left middle cerebral artery occlusion (MCAO) in rats using the intraluminal filament technique as described previously [19]. Briefly, the left common carotid artery was exposed and a silicon-coated 4-0 nylon filament was introduced into the left internal carotid artery through the external carotid artery. The intraluminal suture was carefully withdrawn to establish reperfusion after 90 minutes of ischemia to establish transient MCAO model. For permanent MCAO model, the intraluminal suture was not withdrawn. The sham group received similar surgical procedures without occlusion of the middle cerebral artery. Laser Doppler (Perimed, Jarfalla, Sweden) was used to monitor cerebral blood flow $(\mathrm{CBF})$ during the procedure to confirm occlusion and excluded the rats with a decline rate less than $70 \%$. Body temperature was monitored by a rectal probe and maintained at $37^{\circ} \mathrm{C}$ using a feedback-regulated heating system (CMA/Micro Dialysis AB, Knivsta, Sweden) during surgery.

2.4. Drug Administration. The rats were randomly divided into the following 6 groups: sham group, model group, Xueshuantong $25 \mathrm{mg} / \mathrm{kg}$ group (XST-25), Xueshuantong $50 \mathrm{mg} / \mathrm{kg}$ group (XST-50), Xueshuantong $100 \mathrm{mg} / \mathrm{kg}$ group (XST-100), and edaravone group (as a positive control drug, $6 \mathrm{~mL} / \mathrm{kg}$ ). $50 \mathrm{mg} / \mathrm{kg}$ dose was converted from a commonly used dosage of XST in clinical practice in our study.

Experiment I. To examine the acute neuroprotection of Xueshuantong on transient MCAO and the mechanism of this effect, Xueshuantong was administrated i.v. immediately after ischemia in transient MCAO rats once a day continuously for 1 or 3 days with 6 groups: sham group ( $n=9$, none died), model group $(n=20,3$ died $), \operatorname{XST}-25(n=$ 10,2 died), XST-50 ( $n=10,2$ died), XST-100 $(n=21$, 4 died), and edaravone group ( $n=9,1$ died). There was no statistical difference on mortality between these groups. Experiment I assessed infarct volume, neurological score, hemispheric swelling, mRNA expressions of cytokines, and signaling pathway at $24 \mathrm{~h}$ or $72 \mathrm{~h}$ after tMCAO.

Experiment II. To test the effect of the medicine in permanent cerebral ischemia model, which is more clinically meaningful than transient cerebral ischemia model [20], Xueshuantong was administrated i.v. 4 hours after ischemia in permanent MCAO rats with 4 groups: model group $(n=11)$, XST$25(n=11)$, XST-50 $(n=12)$, and XST-100 $(n=11)$. Eight rats survived in all groups. There was no statistical difference in mortality between these groups. Experiment II measured infarct volume and hemispheric swelling at $24 \mathrm{~h}$ after pMCAO.

Experiment III. To assess the effect of Xueshuantong on longterm functional outcomes, Xueshuantong was administrated 
i.v. 4 hours after ischemia in transient MCAO rats once a day continuously for 14 days with 2 groups: model group $(n=$ $12)$ and XST-100 $(n=12)$. The survival rates at $1,3,7,14$, and $28 \mathrm{~d}$ after tMCAO were shown in Section 3.5. Experiment III identified a batch of functional outcomes at 1-28 d after surgery.

2.5. Neurological Deficits. The neurological score was used to assess general neurological status [21]. Tests were performed at 24 and 72 hours after surgery in Experiment I and days 1, $3,7,14$, and 28 after surgeries in Experiment III. The test was scored as follows: 0 , no observable deficit; 1 , forelimb flexion; 2 , decreased resistance to lateral push (and forelimb flexion) without circling; 3 , same behavior as 2 but with circling.

2.6. Determination of Infarct Volume and Swelling Percent by TTC Staining. After 72 hours of ischemia in Experiment I and $24 \mathrm{~h}$ in Experiment II, the rats were deeply anesthetized and brains were rapidly removed, frozen at $-20^{\circ} \mathrm{C}$ for 15 minutes. Seven sections ( $2 \mathrm{~mm}$ thick) were cut using a rodent brain matrix and were stained with $2 \%(\mathrm{w} / \mathrm{v})$ 2,3,5-triphenyltetrazolium chloride (TTC; Sigma, St. Louis, MO, USA). Infarct volume was analyzed with ImageJ software (Wayne Rasband, National Institutes of Health, USA). Swelling percent was calculated with the formula: swelling percent $(\%)=$ (ipsilateral ischemic hemisphere volume - contralateral ischemic hemisphere volume)/contralateral ischemic hemisphere volume $\times 100 \%$.

2.7. Quantitative Real-Time Polymerase Chain Reaction. 72 hours after MCAO, rats of sham, model, and XST-100 group ( $n=6$, resp.) in Experiment I were deeply anesthetized and perfused through the heart with cold PBS. All infarct area and penumbra were taken and mixed. Total RNA was isolated using Trizol reagent and processed for cDNA, followed by quantitative real-time polymerase chain reaction (PCR) as described previously [22] The specific primer pairs (Sangon Technology Co. Ltd., Shanghai, China) are listed in Table 1. The mRNA levels of inflammatory mediators were normalized to the value of GAPDH, and the results were expressed as fold change of the threshold cycle $(\mathrm{Ct})$ value relative to sham-operated controls using the $2^{-\Delta \Delta \mathrm{Ct}}$ method.

2.8. Western Blot Analysis. Twenty-four hours after MCAO, rats of sham, model, and XST-100 group ( $n=3$, resp.) in Experiment I were deeply anesthetized and perfused through the heart with cold PBS. Then protein was extracted and concentration was determined. Samples were electrophoresed in SDS/PAGE gels and transferred onto a PVDF membrane and incubated overnight at $4^{\circ} \mathrm{C}$ with appropriate primary antibody of p38, phospho-p38, ERK, phosphoERK, JNK, phospho-JNK, STAT3, phospho-STAT3, Prx6, TLR4, and $\beta$-actin (Cell Signaling, USA). After incubation with horseradish peroxides' conjugated secondary antibodies (Zhongshan-Golden Bridge, China) for $1 \mathrm{~h}$ at room temperature, the blots were developed with chemiluminescence reagent using an ECL kit (Millipore, USA).
TABLE 1: The specific primer pairs used in polymerase chain reaction.

\begin{tabular}{lcl}
\hline Gene & Accession number & $\begin{array}{l}\text { Primer pair }\left(5^{\prime}-3^{\prime}\right) \text {; F: forward; R: } \\
\text { reverse }\end{array}$ \\
\hline GAPDH & NM017008.4 & $\begin{array}{l}\text { F: CCCCCAATGTATCCGTTGTG } \\
\text { R: TAGCCCAGGATGCCCTTTAGT }\end{array}$ \\
\hline IL-1 $\beta$ & M98820.1 & $\begin{array}{l}\text { F: GAAGTCAAGACCAAAGTGG } \\
\text { R: TGAAGTCAACTATGTCCCG }\end{array}$ \\
\hline IL-23p19 & NM130410.2 & $\begin{array}{l}\text { F: AAAGGAGGTTGATAGAGGGT } \\
\text { R: TCTTAGTAGATCCATTTGTCCC }\end{array}$ \\
\hline IL-17 & NM001106897.1 & $\begin{array}{l}\text { F: CACAAGCTCATCCCGTACCA } \\
\text { R: CAGGCACATGGATGGAATTCT }\end{array}$ \\
\hline TNF $\alpha$ & HQ201305.1 & $\begin{array}{l}\text { F: TCTTCTCATTCCTGCTCGTGG } \\
\text { R: GGTCTGGGCCATGGAACTGA }\end{array}$ \\
\hline iNOS & XM003750865.2 & $\begin{array}{l}\text { F: AAAATGGTTTCCCCCAGTTC } \\
\text { R: GTCGATGGAGTCACATGCAG }\end{array}$ \\
\hline
\end{tabular}

2.9. Functional Assessment. All rats in Experiment III received EBST on days 1, 3, 7, 14, and 28 after surgery, and Forelimb Placing test on days 3, 7, 14, and 28 after surgery, and Foot Fault, Rotarod, and Y-maze test on day 28 after surgery in a blinded manner. Two hours before surgery, rats were assessed to obtain preinjury baselines.

Elevated Body Swing Test (EBST). The swing test is a simple and easy behavioral test that only requires handling the animal by its tail and recording the direction of swings made by the animal for a certain period of time as described previously [23]. A swing was recorded whenever the animal moved its head out of the vertical axis more than $10^{\circ}$ to either side.

Forelimb Placing Test. Independent testing of each forelimb for placing in response to visual, vibrissae, tactile, and proprioceptive stimulation is conducted using a test designed by De Ryck et al. [24]. The number of completed placing responses out of 3 for each test was recorded. Average placing scores of testing were calculated for each animal.

Foot Fault Test. The Foot Fault test has been shown to be a sensitive indicator for detecting impairments of sensorimotor function after ischemia in rodents and requires very little pretraining. Briefly, an animal is placed on an elevated, leveled grid with openings. Each time a paw slips through an open grid, a "foot fault" is recorded [25]. The number of ipsilateral faults for each limb was recorded.

Rotarod. This Rotarod test was to detect motor ability. The rat was practiced for 60 seconds at $4 \mathrm{rpm}$ and test sessions consisted of 3 trials at $10 \mathrm{rpm}$ [26]. If the rats managed to maintain their balance for 300 seconds, the trial was ended. The final score was expressed as the mean time that a rat was able to remain on the rod for the 3 trials. 
Y-Maze. Y-maze is used as a measure of working spatial memory to assess spontaneous alternation. Briefly, each rat was placed at the end of one arm and allowed to move through the maze for 8 minutes. The percentage of alternation was counted [27].

2.10. Statistics. For statistical analysis, a standard software package (SPSS for Windows 19) was used. All data were given as mean \pm standard error (SE). For survival data, which is count data, Fisher's exact test was used. Except for survival rate, all other data were examined for assumption of normality using the Kolmogorov-Smirnov test and for homogeneity of variance using Levene's test. For the data with normality and homogeneity of variance, independentsamples $t$-tests (two-group comparison) or one-way ANOVA with Dunnett's posttests (multigroup comparisons) were used. For nonnormal or unequal variances data, MannWhitney $U$ tests were performed. $P$ values $<0.05$ were considered significant.

\section{Results}

3.1. XST Treatment Decreased Cerebral Infarct Size and Swelling Percent and Improved Neurological Deficits in Transient MCAO Rats. Firstly, we evaluated the effects of XST i.v. immediately after ischemia in transient MCAO rats by cerebral infarct volume, swelling percent, and neurological deficits (Figure 1(a)). Laser Doppler flowmetry was used to measure cerebral blood flow during ischemia and reperfusion in transient MCAO rats (Figure 1(b)). Representative photographs of TTC-stained coronal brain sections that show viable (red) and dead (white) tissue $72 \mathrm{~h}$ after MCAO were shown in Figure 1(c). Compared with the vehicle-treated model group, XST-50, XST-100, and edaravone group showed notably smaller infarct volumes (Figure $1(\mathrm{~d})$ ) and swelling percent (Figure 1(e)). The neurobehavioral tests revealed that XST-100 and edaravone group could also reduce the neurological scores compared with the model group, but without statistical difference (Figure 1(f)), in day 3 after surgery. Edaravone was regarded as positive control drug which has been shown to have neuroprotective effects in animal MCAO experiments [28].

3.2. XST Treatment Inhibited $m R N A$ Expressions of Inflammatory Cytokines in Transient MCAO Rats. We further observed the changes in mRNA levels of interleukin-1 $\beta$ (IL-1 $\beta$ ), IL17 , IL-23p19, tumor necrosis factor- $\alpha(\mathrm{TNF} \alpha)$, and inducible nitric oxide synthase (iNOS) in brain by real-time PCR analysis. Our results showed that ischemia attack significantly upregulated the mRNA levels of IL-1 $\beta$, IL-17, IL-23p19, TNF $\alpha$, and iNOS, respectively. XST treatment reversed the increase of mRNA levels of these cytokines (Figure 2).

3.3. XST Treatment Inhibited the Expression of Prx6-TLR4 Signaling Pathway and Upregulated the Phosphorylation Level of STAT3 in Transient MCAO Rats. As shown in Figure 3, the expression levels of Prx6 and TLR4 in transient MCAO rats were markedly increased in the model group compared with those in the sham groups, while XST treatment could decrease the expression levels of Prx6 and TLR4 compared with model groups $24 \mathrm{~h}$ after MCAO. To elucidate the mechanism of the neuroprotective effects of XST against ischemia brain injury, we further explored the effect of XST on the MAPK signaling pathway in transient MCAO rat brain. As shown in Figure 4, cerebral ischemia significantly induced the subsequent activation of TLR4 signaling effectors, reflected by increasing the phosphorylation levels of p38, ERK, and JNK in the ischemic brain in model group compared with control groups. XST-100 inhibited the phosphorylation level of p38. Phosphorylation of STAT3 is reported to reduce the inflammatory injury through inhibition of Ras-MAPK pathway activation [29]. As shown in Figure 4, the phosphorylation level of STAT3 in the ischemic brain in model group was upregulated. XST could increase the phosphorylation level of STAT3 compared with model groups.

3.4. XST Treatment Decreased Cerebral Infarct Size and Swelling Percent in Permanent MCAO Rats. Next, we evaluated the brain infarct volume and swelling percent in permanent MCAO rats. XST was administrated i.v. at $4 \mathrm{~h}$ after ischemia in permanent MCAO rats, which were sacrificed at $24 \mathrm{~h}$ after ischemia (Figure 5(a)). Representative photographs of TTC-stained coronal brain $24 \mathrm{~h}$ after MCAO were shown in Figure 5(b). Treatment of XST significantly decreased the infarct volume (Figure 5(c)) and swelling percent (Figure 5(d)) in a dose-dependent manner.

3.5. XST Treatment Improved Long-Term Functional Outcome in Transient MCAO Rats. Six functional assessments, including neurological score, Forelimb Placing test, EBST, Rotarod test, Foot Fault test, and Y-maze test, were performed at days $1,3,7,14$, and 28 after surgeries to evaluate long-term functional outcome of the treatment of XST (Figure 6(a)). XST-100 could significantly increase body weight after being administrated at 1 and 7 days after MCAO compared with model group (Figure 6(b)). XST-100 could decrease neurological score on days 7 and 14 after surgery (Figure 6(c)), improve Forelimb Placing test score on days 3 and 28 (Figure 6(e)), and lengthen movement time on day 28 (Figure 6(f)). Compared with model group, XST-100 group showed no significant change in EBST (Figure 6(d)), Foot Fault test (Figure 6(g)), Y-maze test (Figure 6(h)), and survival rate (Figure 6(i)).

\section{Discussion}

Stroke is a serious disease for human being. There is a great demand for intervention therapy. Unfortunately, although more than 700 drugs that target cerebral ischemia showed beneficial effects in preclinical animal studies, none of them proved efficacious in treating stroke patients in the past several decades [30]. This implies to us that one-target treatment for stroke was impossible [31, 32]. Meanwhile XST is a natural mixture, extracted from San-chi, composed of several components, such as ginsenosides Rbl, Rgl, Rd, Re, 

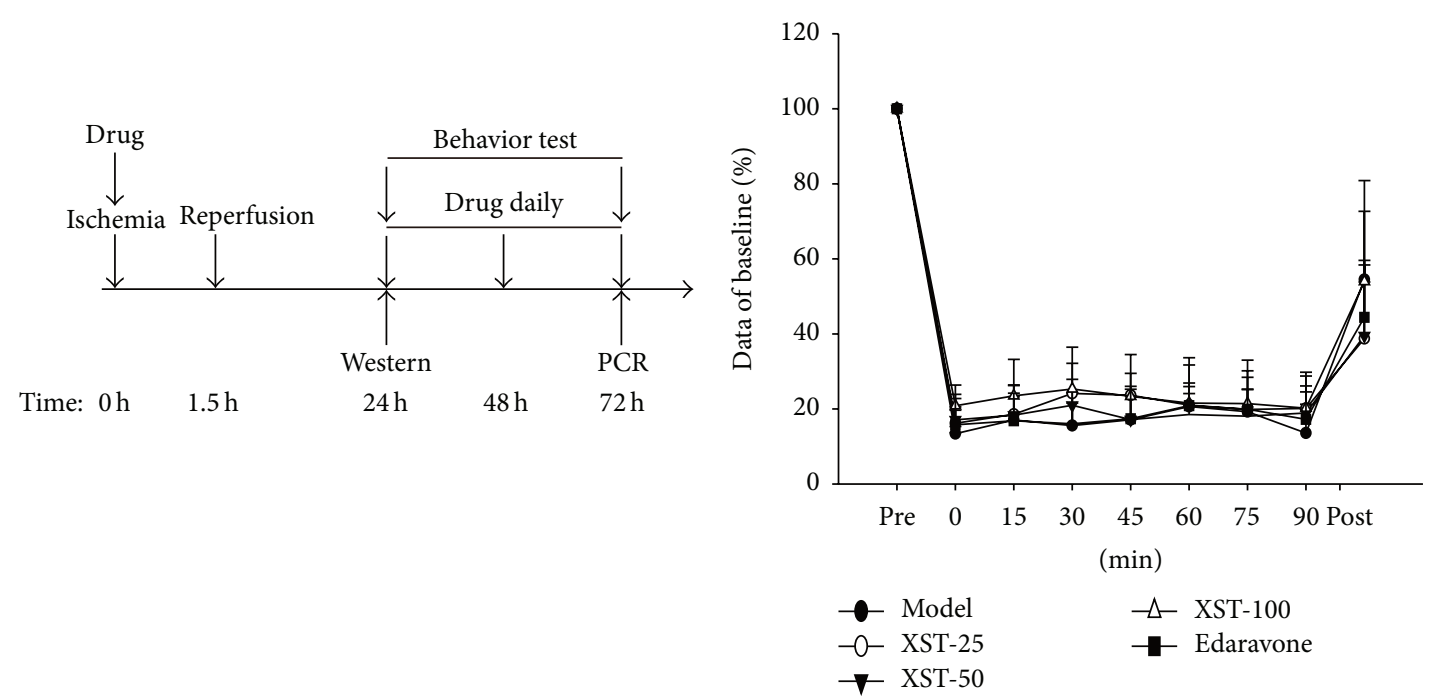

(a) Experimental protocol

(b) Blood flow

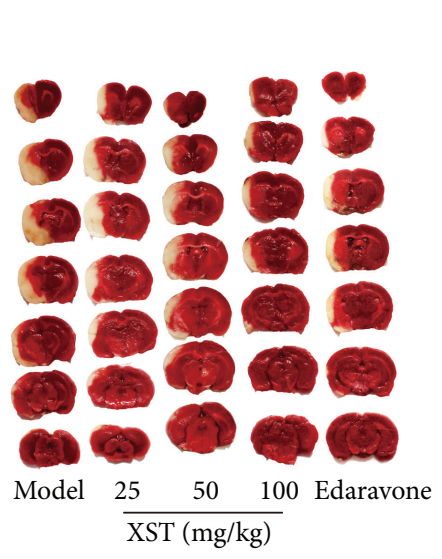

(c) Representative TTC staining

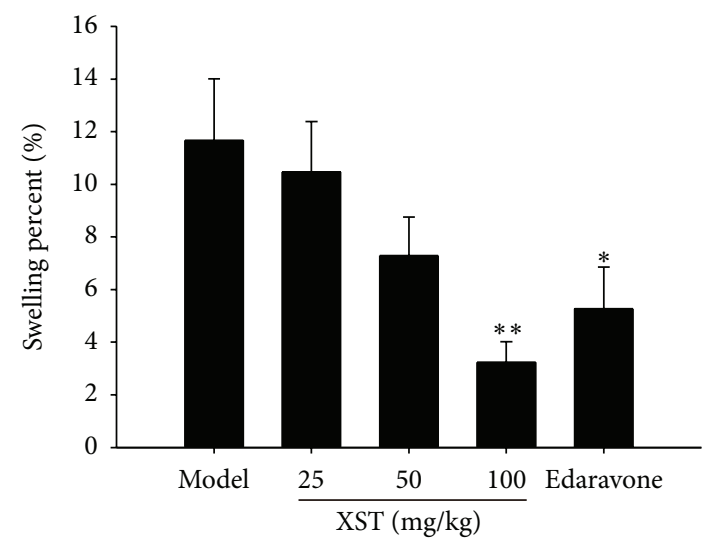

(e) Swelling percent

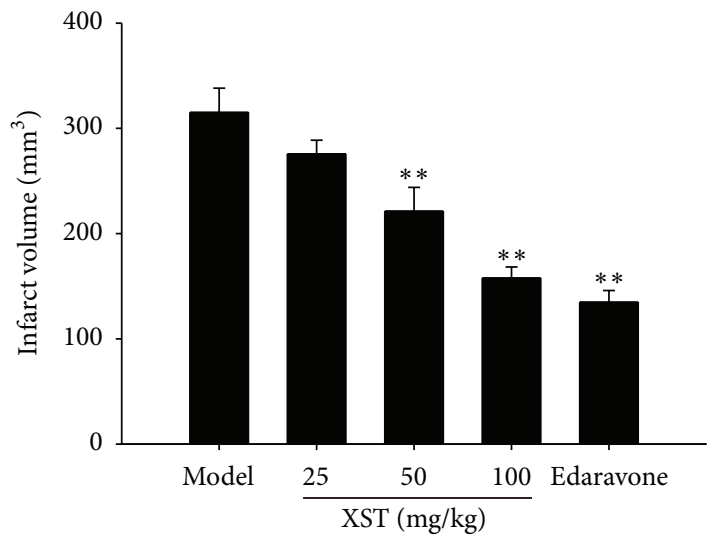

(d) Infarct volume

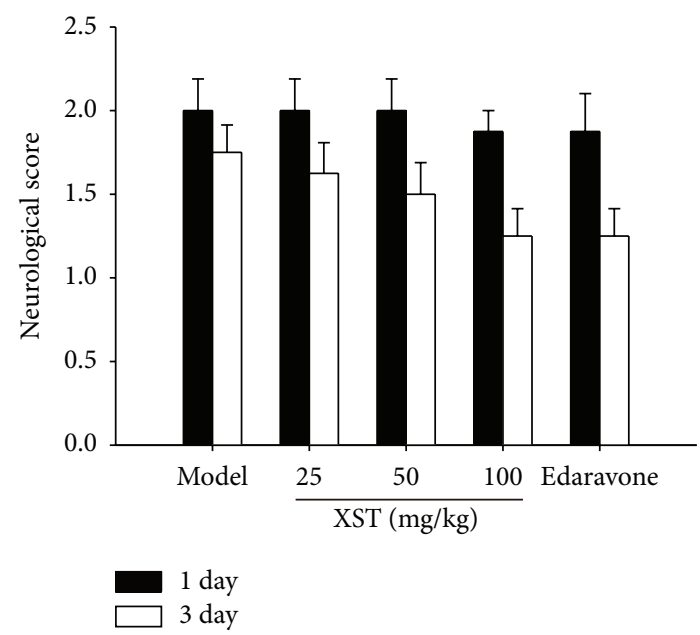

(f) Neurological score

FIGURE 1: Administration with XST immediately after ischemia attenuated cerebral infarct volume and swelling percent and improved behavioral score in transient MCAO rats. (a) The experiment I protocol. (b) Blood flow of rat brain during the surgery. (c) Representative photographs of TTC-stained coronal brain sections that show viable (red) and dead (white) tissue $72 \mathrm{~h}$ after surgery. (d) Cerebral infarct volume expressed as whole infarct area. (e) Swelling percent expressed as a percentage of contralateral brain. (f) Neurological score of rat on days 1 and 3 after surgery. $n=8 .{ }^{*} P<0.05,{ }^{* *} P<0.01$, compared with model group. 

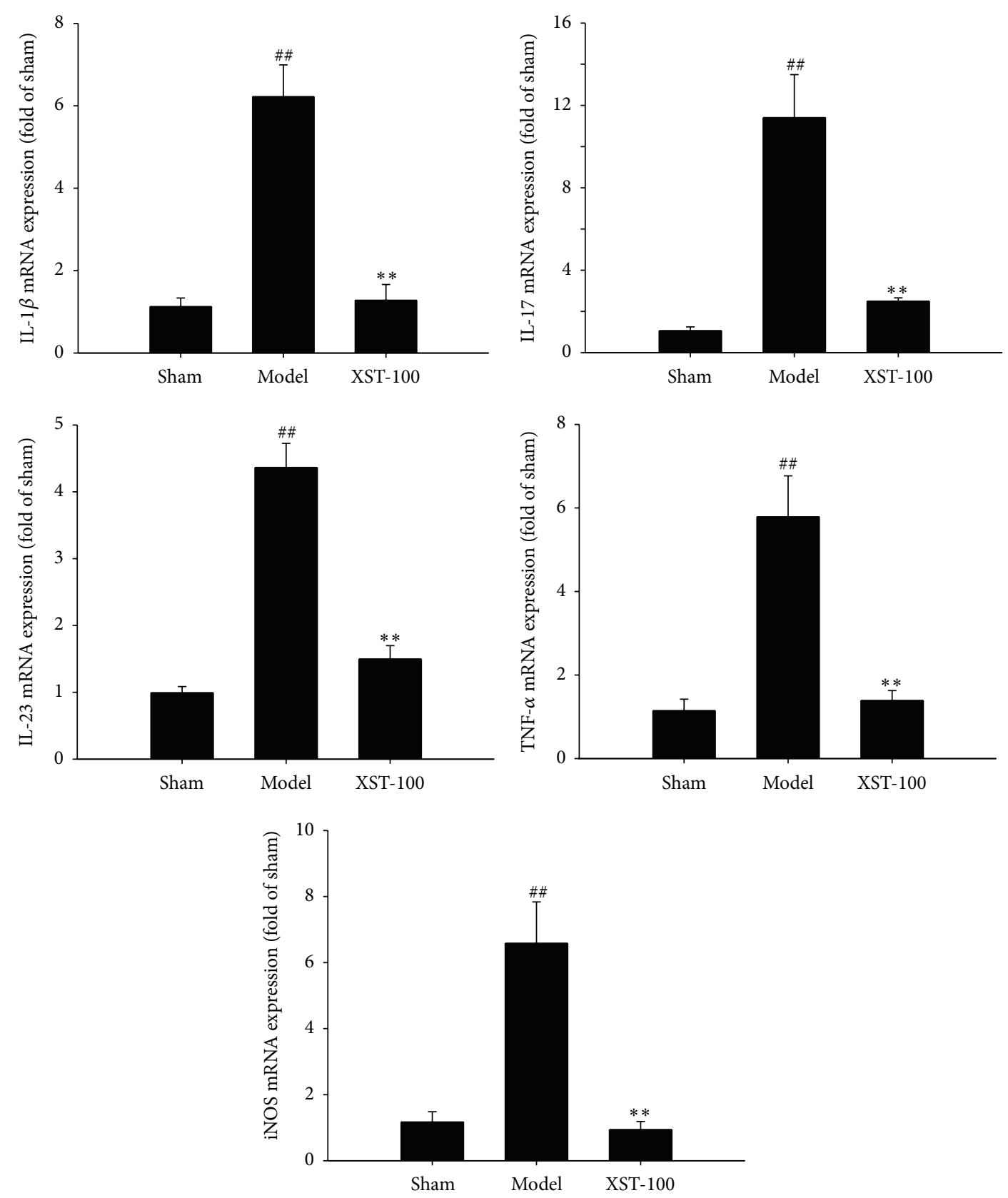

FIGURE 2: Administration with XST immediately after ischemia (the experiment I protocol) decreases the mRNA expressions of IL-1 $\beta$, IL-17, IL-23p19, TNF $\alpha$, and iNOS in ipsilateral ischemic hemisphere. $n=6 .{ }^{\# \#} P<0.01$, compared with control group; ${ }^{* *} P<0.01$, compared with model group.

and $P$. notoginseng saponin R1. It is reported that these components have showed neuroprotection through a variety of mechanisms. Rb1 can weaken the activity of microglia, decrease the upregulation of brain tissue mRNA of TNF $\alpha$ and interleukin-1 (IL-1), IL- $\beta$, and IL-6, and decrease expression of cox- 2 mRNA and protein content in the brain induced by systemic lipopolysaccharide (LPS) treatment in C57BL/6 mice [33]. Rg1 can decrease the expression of c-fos gene and protein in the hippocampus of aged rats [34] and improve the learning and memory function of rats with electrical hippocampal injury [35]. Ginsenoside Rd can attenuate redox imbalance and improve stroke outcome after focal cerebral ischemia in aged mice and attenuate mitochondrial dysfunction and sequential apoptosis after transient focal ischemia by reducing inflammatory response and protecting mitochondria [36, 37]. Ginsenoside Re can reduce the MDA content and the apoptosis of $\mathrm{H}^{+}$-ATP to protect the neurons from ischemia injury in MCAO rats [38]. P. notoginseng saponin 

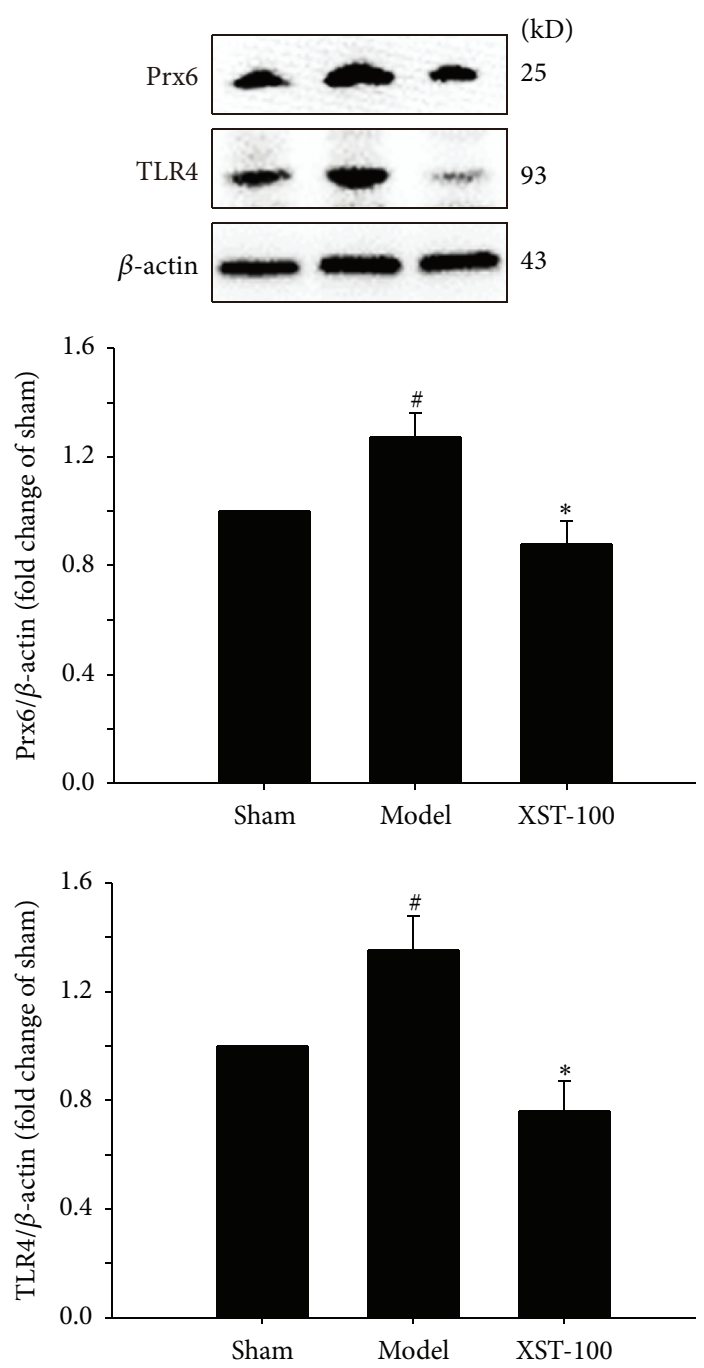

FIGURE 3: Administration with XST immediately after ischemia (the experiment I protocol) reduced the expression of TLR4 and Prx6 in ipsilateral ischemic hemisphere. Representative Western blots and quantitative analysis of Prx6 and TLR4 expression in the ischemic brain tissues $24 \mathrm{~h}$ after transient MCAO. The ratio of Prx6 and TLR4 to $\beta$-actin was densitometrically analyzed and expressed as the relative optical density of the sham group. $n=3$. ${ }^{\#} P<0.05$, compared with control group; ${ }^{*} P<0.05$, compared with model group.

R1 can protect viability of rat neural stem cells and neuronal cells from glutamate interference in primary cultured mouse cortical neurons [39]. Based on these bioactive compounds, we suppose that XST could prevent against ischemic stroke through various ways, although our study just explored one signaling pathway.

Most of the time, many studies choose infarct volume to evaluate the neuroprotective effect of a drug, ignoring the long-term assessment, which leads to a failure in translation to clinical practice. In the present study, we explored the neuroprotective effect of XST in the acute and long-term functional outcome after ischemia. Our data showed that treatment with XST immediately after ischemia resulted in a significantly smaller infarct volume and less swelling percent than vehicle-treated model rats in transient MCAO. Importantly, treatment with XST 4 hours after onset of ischemia reduced infarct size and swelling percent in permanent MCAO rats. What is more, functional recovery is the final aim of treatment and we chose a batch of functional assessments to evaluate functional recovery. We found that administration of XST continuously for 14 days improved long-term functional outcome compared with model group. Our results also showed that although $50 \mathrm{mg} / \mathrm{kg}$ dose of XST was converted from a commonly used dosage of XST in clinical practice, XST-100 was the most effective volume in our animal experiment. It suggested that the dose can be raised for a better prognosis. By the way, to confirm our MCAO model and experimental system, we used edaravone as a positive control, which has been proved to be neuroprotective in several rodent MCAO models by many researchers $[28,40,41]$. Our results were consistent with these previous reports $[28,40,41]$. 

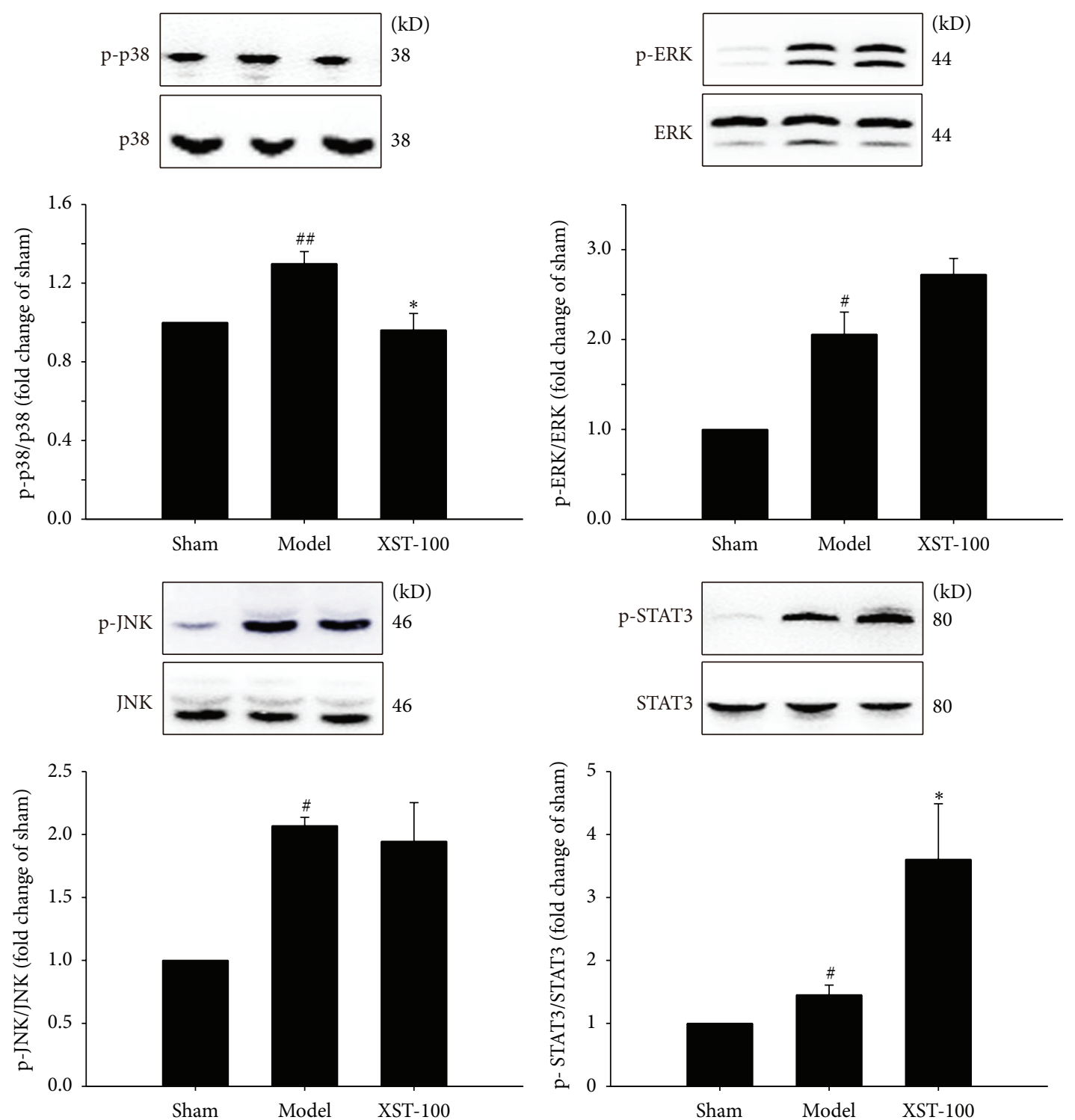

FIGURE 4: Administration with XST immediately after ischemia (the experiment I protocol) reduced the phosphorylation level of p38 and increased the phosphorylation level of STAT3 in transient MCAO rat brain. Representative Western blots and quantitative analysis of phosphorylation levels of p38, ERK, JNK, and STAT3 in the ischemic brain tissues $24 \mathrm{~h}$ after MCAO. The phosphorylation levels of p38, ERK, JNK, and STAT3 were densitometrically analyzed and expressed as the relative optical density of the sham group. $n=3 .{ }^{\#} P<0.05$, ${ }^{\#} P<0.01$, compared with control group; ${ }^{*} P<0.05$, compared with model group.

More and more evidence suggests that both innate and adaptive immunity, highly regulated by TLR/danger associated molecular patterns (DAMPs) signaling, play an irreplaceable role in the progression of postischemic neuroinflammation and brain injury. The binding of DAMPs to TLRs induces innate immune responses through the activation of TLR signaling effectors, including mitogen activated protein kinase (MAPK), resulting in the production of proinflammatory mediators, such as IL-1 $\beta$, IL-17, IL-23p19, TNF $\alpha$, and iNOS $[42,43]$. Prx's were originally described as antioxidative enzymes within brain cells that exert neuroprotective effects by scavenging reactive oxygen species [44]. However, once released from postischemic dead or dying neural cells, Prx's lose antioxidant capacity, act as DAMPs of TLRs, and induce innate and adaptive immune responses by activating TLR signaling, triggering neuroinflammation and brain lesions in ischemic stroke [45]. A recent study identified extracellular Prx 5 and Prx6 as major TLR2/TLR4-dependent DAMPs in aseptic inflammation after cerebral ischemia [46]. Therefore, immunomodulation through TLR/DAMP signaling is a potential therapeutic approach for neuroinflammation and brain injury after ischemic stroke. In this study, we observed the activation of cerebral ischemia induced TLR4 signaling, including enhancing the protein expression of Prx6 


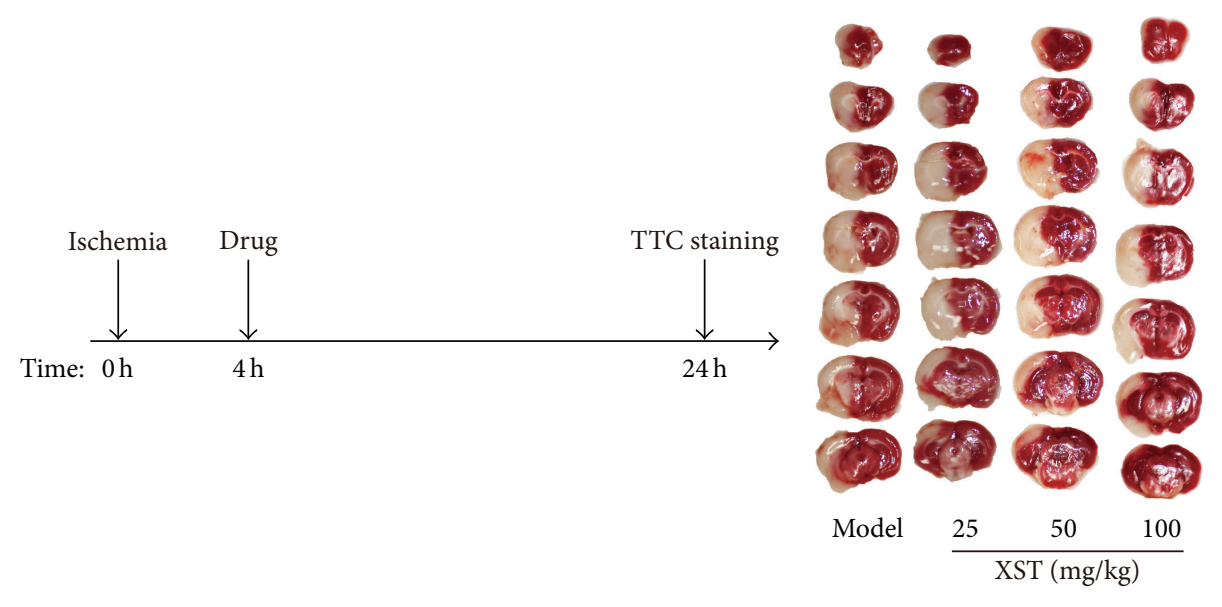

(a) Experimental protocol

(b) Representative TTC staining

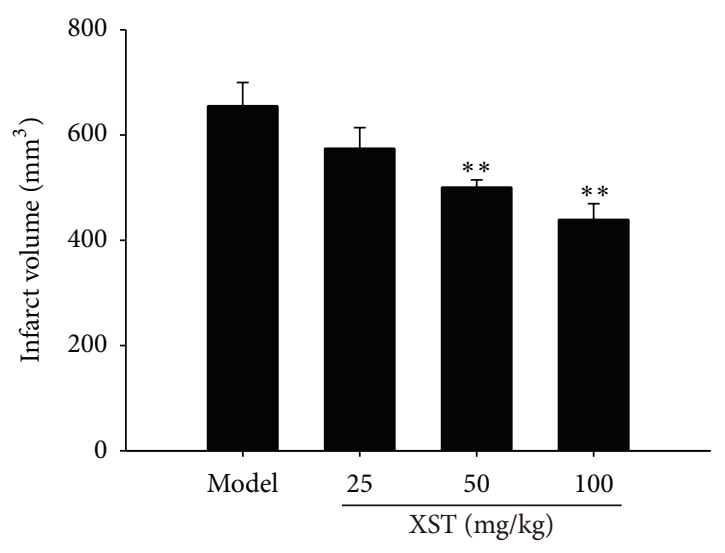

(c) Infarct volume

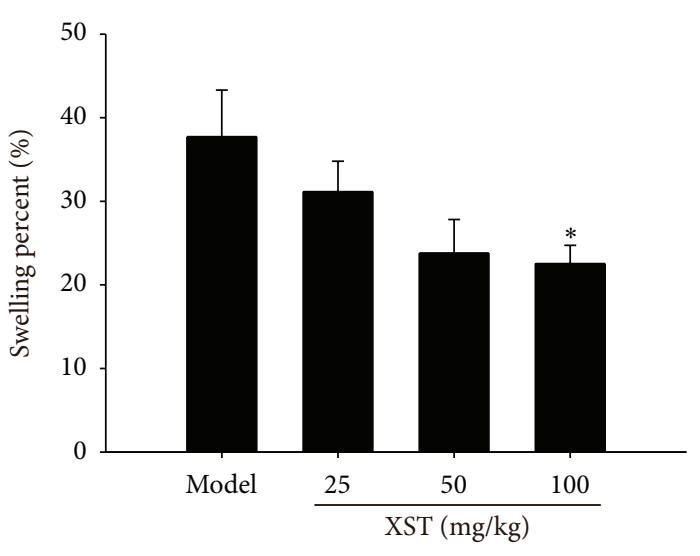

(d) Swelling percent

FIGURE 5: Administration with XST 4 hours after ischemia attenuated cerebral infarct volume and swelling percent in permanent MCAO rats. (a) The experiment II protocol. (b) Representative photographs of TTC-stained coronal brain sections $24 \mathrm{~h}$ after MCAO. (c) Cerebral infarct volume expressed as whole infarct area. (d) Swelling percent expressed as a percentage of contralateral part. $n=8$. ${ }^{*} P<0.05$, ${ }^{* *} P<0.01$, compared with model group.

and TLR4, and the activation of MAPK signal pathway. Administration os XST resulted in less mRNA expression of inflammatory cytokines IL-1 $\beta$, IL-17, IL-23p19, TNF $\alpha$, and iNOS, as well as preventing the upregulation of Prx6, TLR4, and the phosphorylation levels of p38 in transient MCAO rats.

The phosphorylation of STAT3 was reported to suppress the activation of MAPK pathway [29]. Administration of XST could highly increase the phosphorylation levels of STAT3. We suppose that XST could also inhibit MAPK pathway by the phosphorylation of STAT3.

We did not explore intracerebral distribution of XST in this paper. Previous study showed that the original type of panaxadiol saponin of Panax ginseng and protopanaxatriol can be detected in brain after i.v. $(30 \mathrm{mg} / \mathrm{kg})$ administration in vivo, and their concentrations in hippocampal were relatively higher than that in cortex [47]. My team group found that the components of XST were easier to pass blood brain barrier after cerebral ischemia. For example, after administration of XST (i.v., $100 \mathrm{mg} / \mathrm{kg}$ ) $24 \mathrm{~h}$ after surgery, the concentration of ginsenoside Rg1 in ischemic cortex was 15 times in MCAO rats more than in sham group (unpublished).

\section{Conclusions}

In conclusion, our data showed that (1) treatment with XST $(50,100 \mathrm{mg} / \mathrm{kg})$ immediately after ischemia resulted in significant neuroprotection against acute brain injury in a transient MCAO rat model dose-dependently; (2) treatment with XST $(50,100 \mathrm{mg} / \mathrm{kg}) 4$ hours after onset of ischemia reduced infarct size and swelling percent in permanent MCAO rats in a dose-dependent manner; (3) administration of XST $(100 \mathrm{mg} / \mathrm{kg})$ continuously for 14 days improved long-term functional outcome compared with model group. Moreover, the neuroprotective mechanism of XST might be related to 


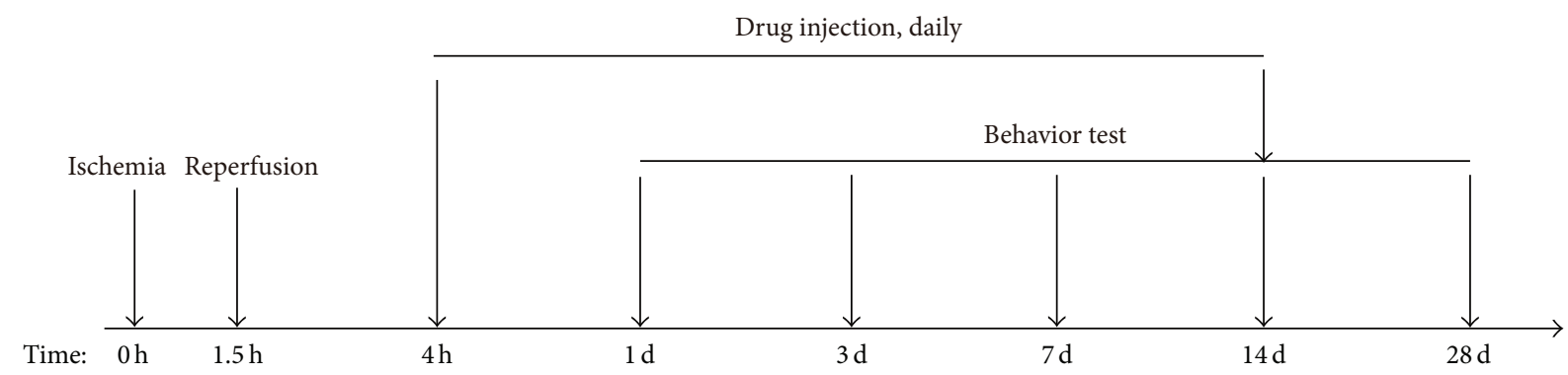

(a) Experimental protocol
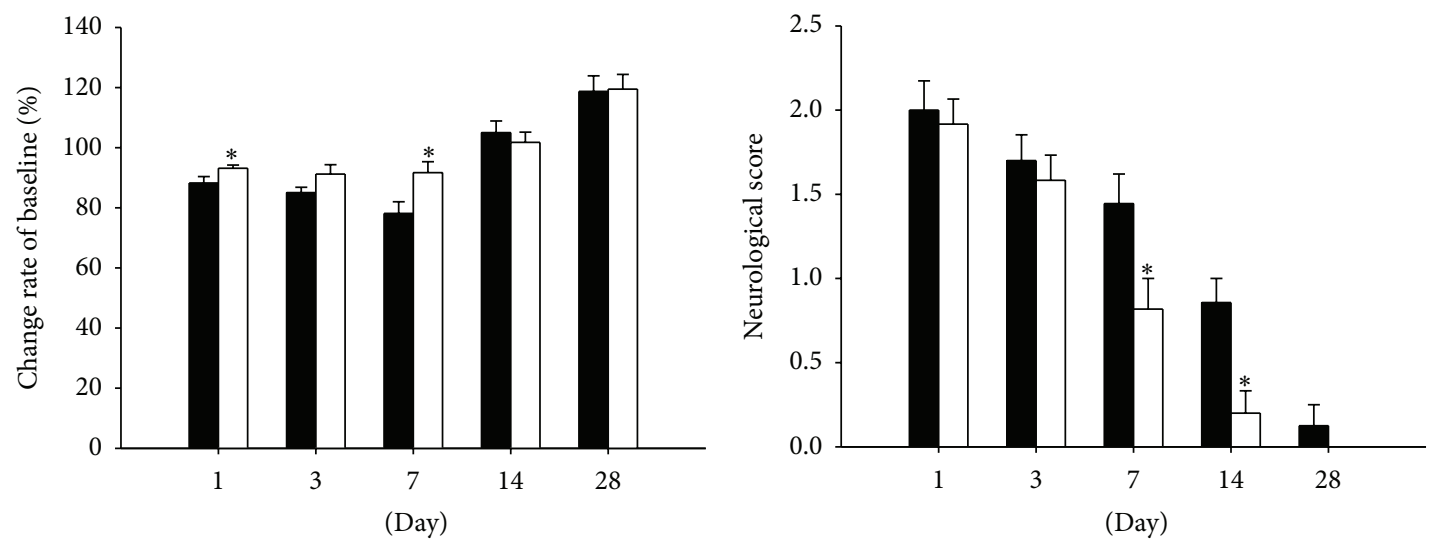

Model
XST-100

(b) Change rate of weight

(c) Neurological score

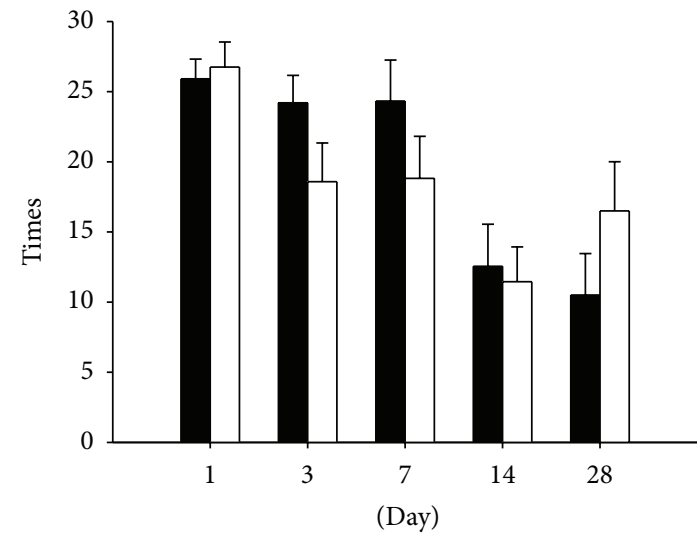

(d) Elevated Body Swing test

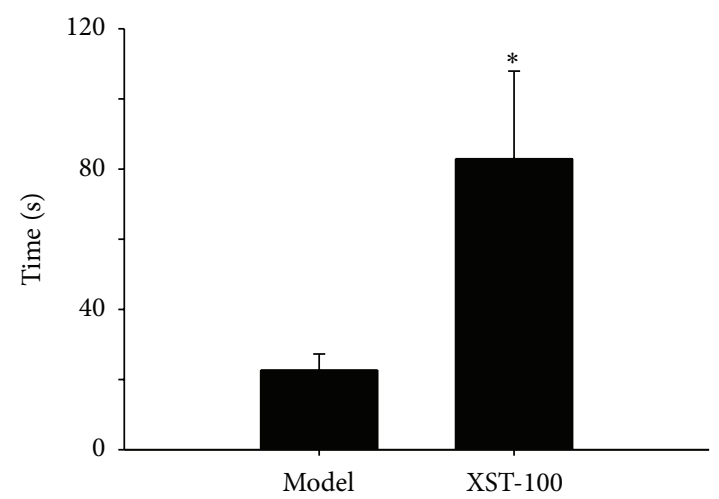

(f) Rotarod

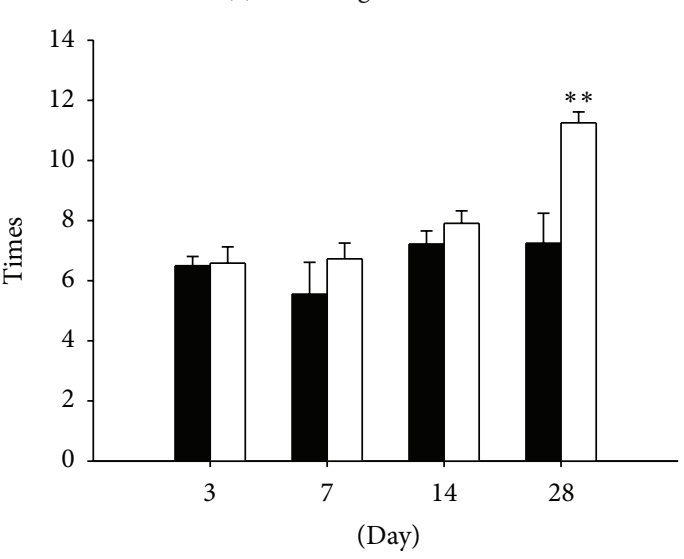

(e) Forelimb Placing test

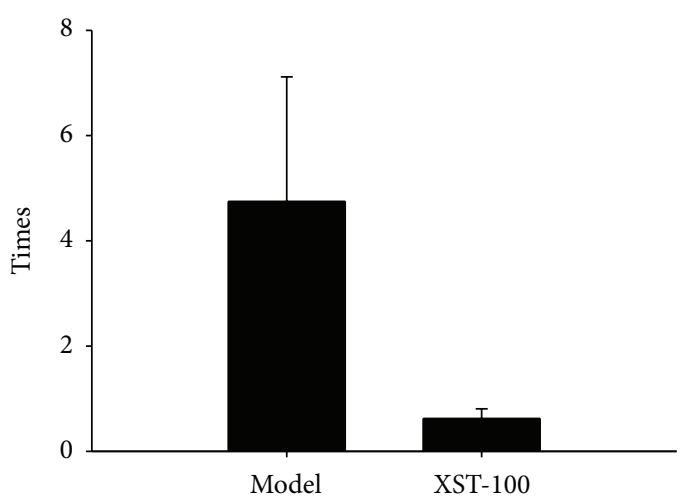

(g) Foot Fault

FIGURE 6: Continued. 


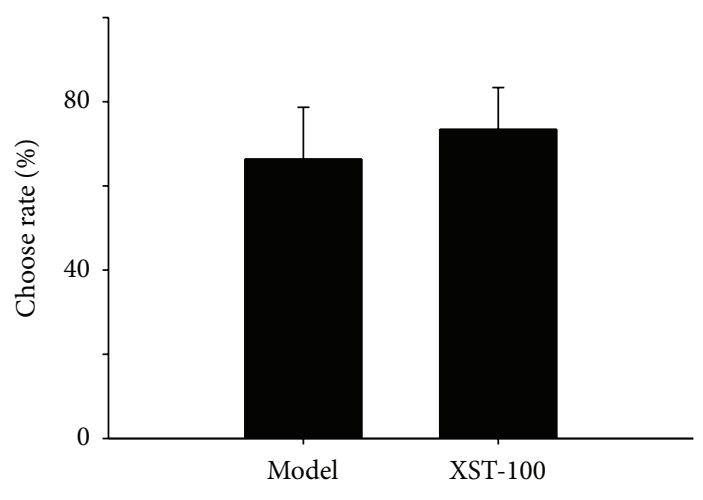

(h) Y-maze

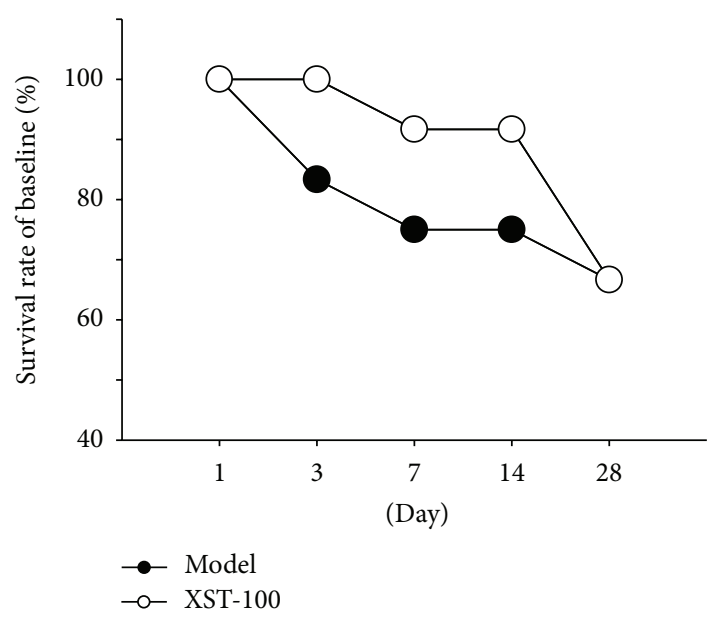

(i) Survival rate of baseline

FIGURE 6: XST treatment improves long-term functional outcome in transient MCAO rats. Administration with XST 4 hours after ischemia improved long-term functional outcome after $90 \mathrm{~min}$ ischemia in transient MCAO rats. (a) The experiment III protocol. (b) Change of weight from days 1 to 28 after surgery. (c) Neurological score from days 1 to 28 after surgery. (d) Elevated Body Swing test (EBST) from days 1 to 28 after surgery. (e) Forelimb Placing test from days 3 to 28 after surgery. (f) Rotarod test on day 28 after surgery. (g) Foot Fault test on day 28 after surgery. (h) Y-maze test on day 28 after surgery. (i) Survival rate of baseline from days 1 to 28 after surgery. $n=8-12 .{ }^{*} P<0.05$, ${ }^{* *} P<0.01$, compared with model group.

prevention of expressions of inflammatory cytokines and Prx6-TLR4 pathway.

\section{Disclosure}

Xumei Wang and Shaoxia Wang are equal contributors and co-first authors.

\section{Conflict of Interests}

The authors declare that they have no conflict of interests.

\section{Acknowledgments}

This work was supported in part by Major New Drugs Innovation and Development (2012ZX09101201-004), National Natural Sciences Foundation (81173592), Natural Science Foundation of Tianjin and Guangxi (14JCYBJC28900 and 14125008-2-5), College Academic Innovation Team (TD125035), Program for Changjiang Scholars and Innovative Research Team in University (IRT-14R41), and New Century Excellent Talents in University (NCET-13-0935).

\section{References}

[1] R. Matsuo, M. Kamouchi, T. Ago et al., "Thrombolytic therapy with intravenous recombinant tissue plasminogen activator in Japanese older patients with acute ischemic stroke: Fukuoka Stroke Registry," Geriatrics \& Gerontology International, vol. 14, no. 4, pp. 954-959, 2014.

[2] J. C. Grotta, W. S. Burgin, A. El-Mitwalli et al., "Intravenous tissue-type plasminogen activator therapy for ischemic stroke, Houston experience 1996 to 2000," Archives of Neurology, vol. 58, no. 12, pp. 2009-2013, 2001.
[3] J. De Keyser, M. Uyttenboogaart, M. W. Koch et al., "Neuroprotection in acute ischemic stroke," Acta Neurologica Belgica, vol. 105, no. 3, pp. 144-148, 2005.

[4] D. Brea, M. Blanco, P. Ramos-Cabrer et al., "Toll-like receptors 2 and 4 in ischemic stroke: outcome and therapeutic values," Journal of Cerebral Blood Flow \& Metabolism, vol. 31, no. 6, pp. 1424-1431, 2011.

[5] C. Zuany-Amorim, J. Hastewell, and C. Walker, "Toll-like receptors as potential therapeutic targets for multiple diseases," Nature Reviews Drug Discovery, vol. 1, no. 10, pp. 797-807, 2002.

[6] J. Qiu, M. Nishimura, Y. Wang et al., "Early release of HMGB1 from neurons after the onset of brain ischemia," Journal of Cerebral Blood Flow and Metabolism, vol. 28, no. 5, pp. 927-938, 2008.

[7] T. Shichita, E. Hasegawa, A. Kimura et al., "Peroxiredoxin family proteins are key initiators of post-ischemic inflammation in the brain," Nature Medicine, vol. 18, no. 6, pp. 911-917, 2012.

[8] X. M. Wang, S. X. Wang, and L. M. Hu, "Neuroprotective effect of panax notoginseng saponins and its main components," World Journal of Neuroscience, vol. 4, no. 1, pp. 12-17, 2014.

[9] C. Iadecola and J. Anrather, "The immunology of stroke: from mechanisms to translation," Nature Medicine, vol. 17, no. 7, pp. 796-808, 2011.

[10] Q. Shang, H. Xu, Z. Liu, K. Chen, and J. Liu, "Oral Panax notoginseng preparation for coronary heart disease: a systematic review of randomized controlled trials," Evidence-Based Complementary and Alternative Medicine, vol. 2013, Article ID 940125, 12 pages, 2013.

[11] W. Vongsangnak, J. Gua, S. Chauvatcharin, and J.-J. Zhong, "Towards efficient extraction of notoginseng saponins from cultured cells of Panax notoginseng," Biochemical Engineering Journal, vol. 18, no. 2, pp. 115-120, 2004.

[12] D. Xu, P. Huang, Z. Yu et al., "Efficacy and safety of Panax notoginseng saponin therapy for acute intracerebral hemorrhage, meta-analysis, and mini review of potential mechanisms 
of action," Frontiers in Neuroscience, vol. 7, pp. 274-293, 2015.

[13] K.-T. Choi, "Botanical characteristics, pharmacological effects and medicinal components of Korean Panax ginseng C A Meyer," Acta Pharmacologica Sinica, vol. 29, no. 9, pp. 1109-1118, 2008.

[14] L.-Q. Jin and L. Shi, "Effects of saponins of Panax notoginseng on sodium-potassium-adenosine triphosphatase and calciummagnesium-adenosine triphosphatase," Zhongguo Yao Li Xue Bao, vol. 12, no. 6, pp. 504-506, 1991.

[15] J. Zhu, X. Fan, Y. Cheng et al., "Chemometric analysis for identification of botanical raw materials for pharmaceutical use: a case study using Panax notoginseng," PLoS ONE, vol. 9, no. 1, Article ID e87462, 2014.

[16] Southern Medicine Economic Research Institute, “The 2014 blue book of the development of Chinese pharmaceutical market," Progress in Pharmaceutical Sciences, vol. 38, no. 7, pp. 481-496, 2014.

[17] D. J. Lin, "HPLC fingerprints of freeze-dried xueshuantong for injection," China Pharmacy, vol. 20, no. 33, pp. 2606-2608, 2009.

[18] P. Fu and Z. Y. Liu, "Determination of the effecient elements of Xuesaitong for injection, Xueshuantong injection and Xuemaitong for injection by fingerprint," West China Journal of Pharmaceutical Sciences, vol. 22, no. 1, pp. 76-78, 2007.

[19] M. Sawada, N. J. Alkayed, S. Goto et al., "Estrogen receptor antagonist ICI182,780 exacerbates ischemic injury in female mouse," Journal of Cerebral Blood Flow and Metabolism, vol. 20, no. 1, pp. 112-118, 2000.

[20] M. Fisher, G. Feuerstein, D. W. Howells et al., "Update of the stroke therapy academic industry roundtable preclinical recommendations," Stroke, vol. 40, no. 6, pp. 2244-2250, 2009.

[21] J. B. Bederson, L. H. Pitts, M. Tsuji, M. C. Nishimura, R. L. Davis, and H. Bartkowski, "Rat middle cerebral artery occlusion: evaluation of the model and development of a neurologic examination," Stroke, vol. 17, no. 3, pp. 472-476, 1986.

[22] X. Kuang, Y.-S. Chen, L.-F. Wang et al., "Klotho upregulation contributes to the neuroprotection of ligustilide in an Alzheimer's disease mouse model," Neurobiology of Aging, vol. 35, no. 1, pp. 169-178, 2014.

[23] C. V. Borlongan and P. R. Sanberg, "Elevated body swing test: a new behavioral parameter for rats with 6-hydroxydopamineinduced hemiparkinsonism," The Journal of Neuroscience, vol. 15, no. 7, pp. 5372-5378, 1995.

[24] M. De Ryck, J. Van Reempts, M. Borgers, A. Wauquier, and P. A. J. Janssen, "Photochemical stroke model: flunarizine prevents sensorimotor deficits after neocortical infarcts in rats," Stroke, vol. 20, no. 10, pp. 1383-1390, 1989.

[25] K. L. Schaar, M. M. Brenneman, and S. I. Savitz, "Functional assessments in the rodent stroke model," Experimental and Translational Stroke Medicine, vol. 2, article 13, 2010.

[26] S. Sugiura, K. Kitagawa, S. Tanaka et al., "Adenovirus-mediated gene transfer of heparin-binding epidermal growth factor-like growth factor enhances neurogenesis and angiogenesis after focal cerebral ischemia in rats," Stroke, vol. 36 , no. 4, pp. 859864, 2005.

[27] I. B. Nieradko and A. Borzêcki, "Effect of cypermethrin on memory, movement activity and coordination in mice after transient incomplete cerebral ischemia," Pharmacological Reports, vol. 60, no. 5, pp. 699-705, 2008.

[28] P. Zhang, W. Li, L. Li et al., "Treatment with edaravone attenuates ischemic brain injury and inhibits neurogenesis in the subventricular zone of adult rats after focal cerebral ischemia and reperfusion injury," Neuroscience, vol. 201, pp. 297-306, 2012.

[29] M. Carrithers, S. Tandon, S. Canosa, M. Michaud, D. Graesser, and J. A. Madri, "Enhanced susceptibility to endotoxic shock and impaired STAT3 signaling in CD31-deficient mice," The American Journal of Pathology, vol. 166, no. 1, pp. 185-196, 2005.

[30] Y. C. Chen, J. S. Wu, S. T. Yang et al., "Stroke, angiogenesis and phytochemicals," Frontiers in Bioscience, vol. 4, pp. 599-610, 2012.

[31] G. J. Del Zoppo, "Inflammation and the neurovascular unit in the setting of focal cerebral ischemia," Neuroscience, vol. 158, no. 3, pp. 972-982, 2009.

[32] S. C. Cramer, "Repairing the human brain after stroke: I. Mechanisms of spontaneous recovery," Annals of Neurology, vol. 63, no. 3, pp. 272-287, 2008.

[33] J.-S. Lee, J.-H. Song, N.-W. Sohn, and J.-W. Shin, "Inhibitory effects of ginsenoside Rbl on neuroinflammation following systemic lipopolysaccharide treatment in mice," Phytotherapy Research, vol. 27, no. 9, pp. 1270-1276, 2013.

[34] M. Liu and J.-T. Zhang, "Effects of ginsenoside Rg1 on c-fos gene expression and cAMP levels in rat hippocampus," Zhongguo Yao Li Xue Bao, vol. 17, no. 2, pp. 171-174, 1996.

[35] Z.-Y. Chen, T.-M. Du, and S.-C. Chen, "Effects of ginsenoside Rgl on learning and memory function and morphology of hippocampal neurons of rats with electrical hippocampal injuries," Journal of Southern Medical University, vol. 31, no. 6, pp. 10391042, 2011.

[36] R. Ye, X. Kong, Q. Yang, Y. Zhang, J. Han, and G. Zhao, "Ginsenoside Rd attenuates redox imbalance and improves stroke outcome after focal cerebral ischemia in aged mice," Neuropharmacology, vol. 61, no. 4, pp. 815-824, 2011.

[37] R. Ye, X. Zhang, X. Kong et al., "Ginsenoside Rd attenuates mitochondrial dysfunction and sequential apoptosis after transient focal ischemia," Neuroscience, vol. 178, pp. 169-180, 2011.

[38] L.-M. Chen, X.-M. Zhou, Y.-L. Cao, and W.-X. Hu, "Neuroprotection of ginsenoside Re in cerebral ischemia-reperfusion injury in rats," Journal of Asian Natural Products Research, vol. 10, no. 5, pp. 439-445, 2008.

[39] B. Gu, N. Nakamichi, W.-S. Zhang et al., "Possible protection by notoginsenoside R1 against glutamate neurotoxicity mediated by N-methyl-D-aspartate receptors composed of an NR1/NR2B subunit assembly," Journal of Neuroscience Research, vol. 87, no. 9, pp. 2145-2156, 2009.

[40] K. Kikuchi, K.-I. Kawahara, S. Tancharoen et al., "The free radical scavenger edaravone rescues rats from cerebral infarction by attenuating the release of high-mobility group box-1 in neuronal cells," Journal of Pharmacology and Experimental Therapeutics, vol. 329, no. 3, pp. 865-874, 2009.

[41] K. Okamura, T. Tsubokawa, H. Johshita, H. Miyazaki, and Y. Shiokawa, "Edaravone, a free radical scavenger, attenuates cerebral infarction and hemorrhagic infarction in rats with hyperglycemia," Neurological Research, vol. 36, no. 1, pp. 65-69, 2014.

[42] C. L. Willis, "Glia-induced reversible disruption of bloodbrain barrier integrity and neuropathological response of the neurovascular unit," Toxicologic Pathology, vol. 39, no. 1, pp. 172185, 2011.

[43] M. Lv, Y. Liu, J. Zhang et al., "Roles of inflammation response in microglia cell through Toll-like receptors $2 /$ interleukin23/interleukin-17 pathway in cerebral ischemia/reperfusion injury," Neuroscience, vol. 176, pp. 162-172, 2011. 
[44] J.-H. Yi, S.-W. Park, R. Kapadia, and R. Vemuganti, "Role of transcription factors in mediating post-ischemic cerebral inflammation and brain damage," Neurochemistry International, vol. 50, no. 7-8, pp. 1014-1027, 2007.

[45] J. Rashidian, M. W. Rousseaux, K. Venderova et al., "Essential role of cytoplasmic cdk5 and Prx 2 in multiple ischemic injury models, in vivo," The Journal of Neuroscience, vol. 29, no. 40, pp. 12497-12505, 2009.

[46] K.-S. Yang, S. W. Kang, H. A. Woo et al., "Inactivation of human peroxiredoxin I during catalysis as the result of the oxidation of the catalytic site cysteine to cysteine-sulfinic acid," The Journal of Biological Chemistry, vol. 277, no. 41, pp. 38029-38036, 2002.

[47] L. T. Kong, Pharmacokinetic Study of Ginseng Saponins, Peking Union Medical College, 2013. 


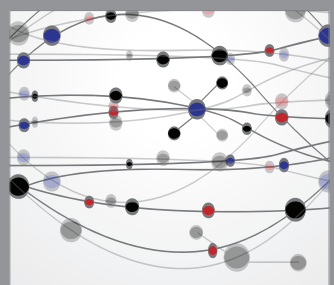

The Scientific World Journal
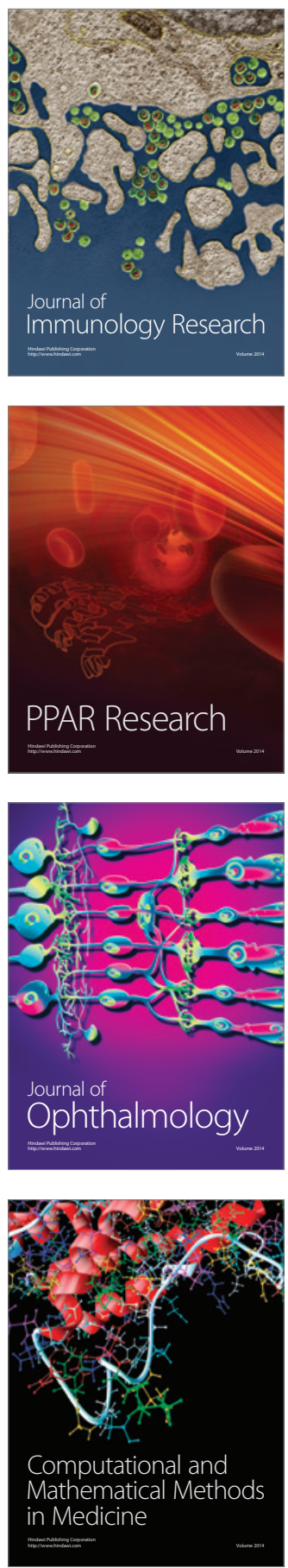

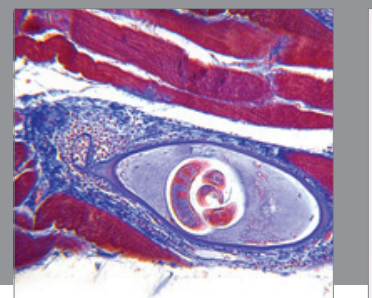

Gastroenterology

Research and Practice
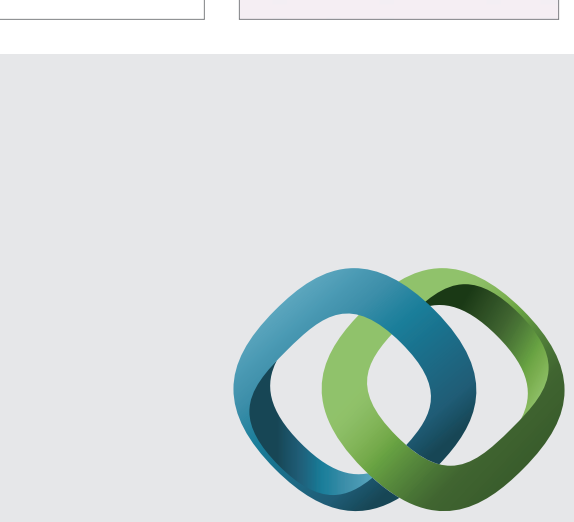

\section{Hindawi}

Submit your manuscripts at

http://www.hindawi.com
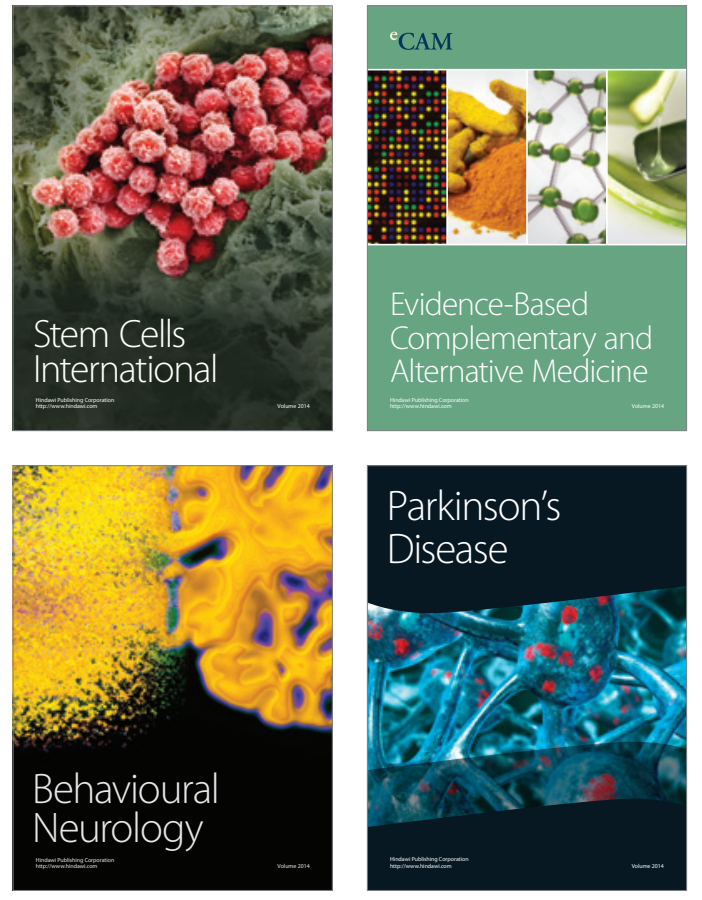
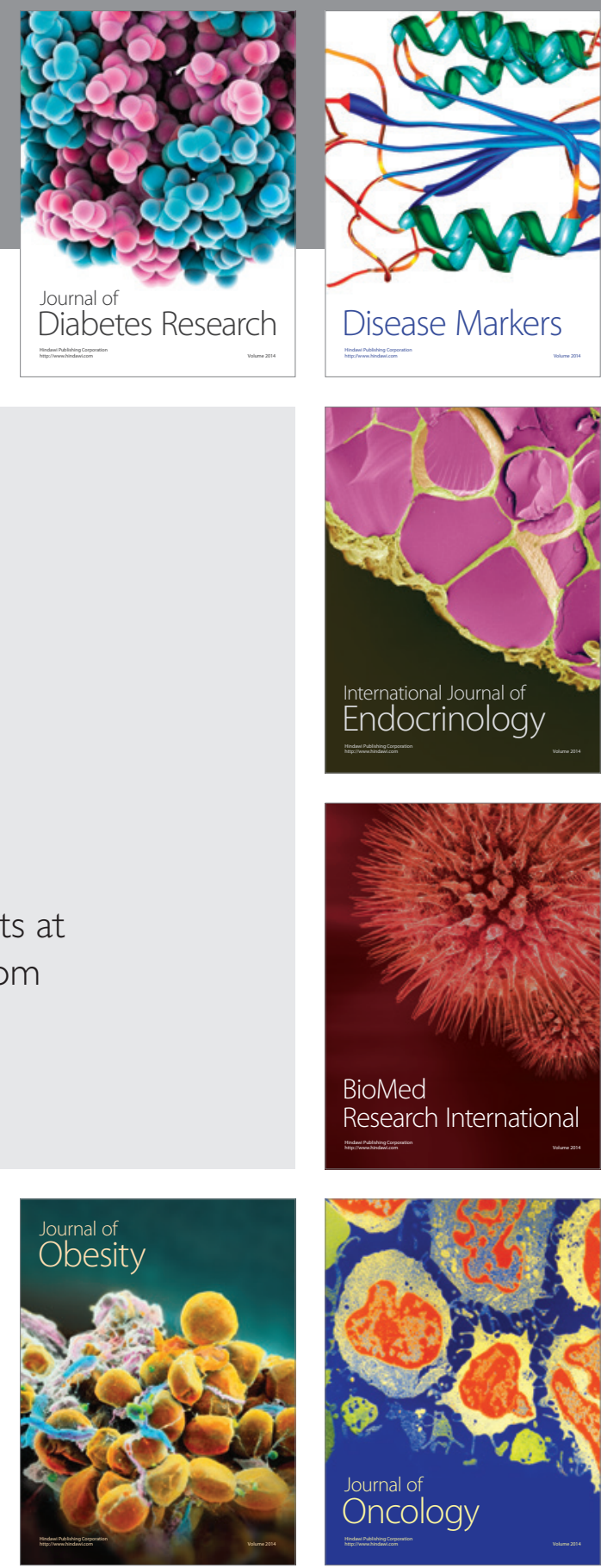

Disease Markers
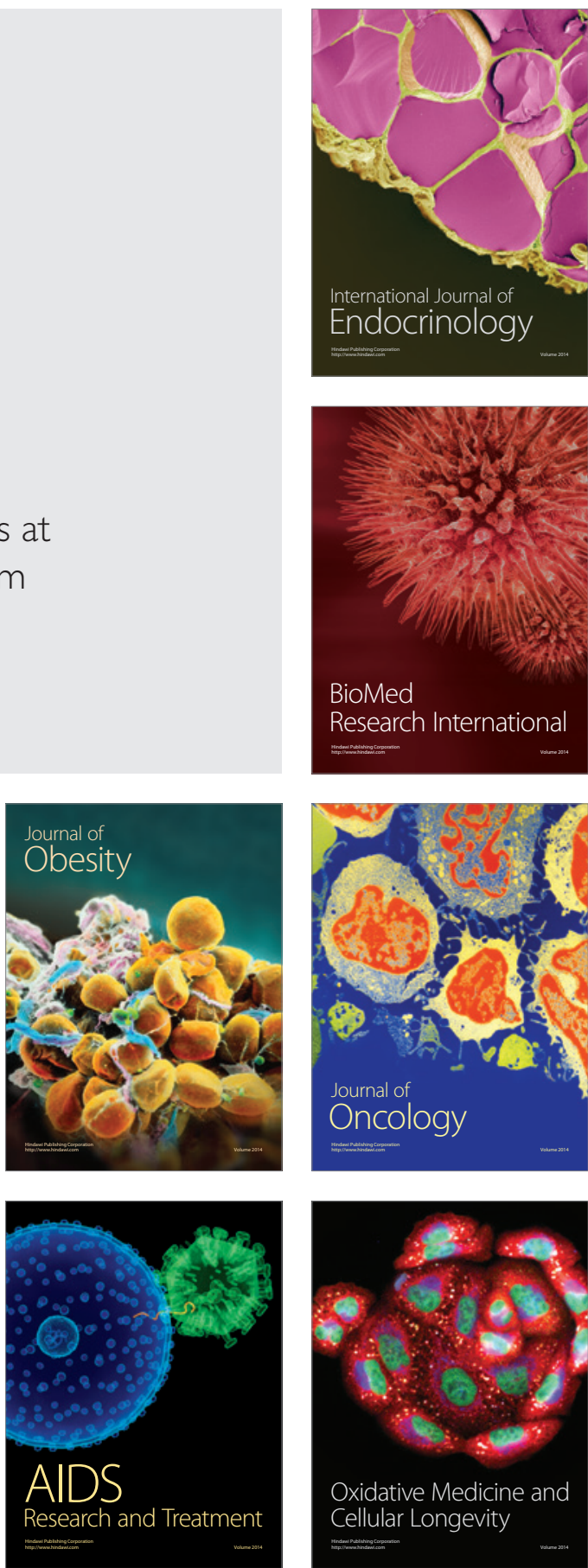